\title{
A Randomised Study to Investigate the Nicotine Pharmacokinetics of Oral Nicotine Pouches and a Combustible Cigarette
}

\author{
Michael McEwan ${ }^{1}$ (1) $\cdot$ David Azzopardi $^{1} \cdot$ Nathan Gale $^{1} \cdot$ Oscar M. Camacho ${ }^{1} \cdot$ George Hardie $^{1} \cdot \operatorname{lan}$ M. Fearon ${ }^{2}$. \\ James Murphy ${ }^{3}$
}

Accepted: 21 November 2021 / Published online: 18 December 2021

(c) The Author(s) 2021

\begin{abstract}
Background and Objectives Nicotine pouches (NPs) are a relatively new type of oral smokeless tobacco-free nicotine product. Currently, few data are available on the nicotine pharmacokinetics or subjective effects of NP use. The objective of this study was to determine and compare the pharmacokinetics of nicotine absorption into the blood from different NP variants and a combustible cigarette.

Methods In a randomised, controlled, crossover clinical study, nicotine pharmacokinetics and subjective effects were compared among commercially available NPs (five different brands; 6-10 mg nicotine/pouch) and a combustible cigarette. During an 8-day confinement period, 35 healthy adult participants who were current dual users of snus and combustible cigarettes used one study product each day for a defined period following overnight nicotine abstinence.

Results Nicotine maximum plasma concentration $\left(C_{\max }\right)$ and area under the plasma concentration-time curve between 0 and $6 \mathrm{~h}\left(\mathrm{AUC}_{0-6 \mathrm{~h}}\right)$ were significantly greater for the Lyft $10 \mathrm{mg} \mathrm{NP}$ than for the cigarette (both $\left.p<0.0001\right)$, while the other NPs had $C_{\max }$ and $\mathrm{AUC}_{0-6 \mathrm{~h}}$ values that were either greater than or similar to those of the cigarette. Plasma nicotine concentration was not associated with the nicotine contents of the NPs. Time to reach maximum plasma concentration $\left(T_{\max }\right)$ was higher for all NPs (60-65 $\mathrm{min}$ ) than for the cigarette $(7 \mathrm{~min})$. Regarding subjective effects, liking and intent to use product again scores were higher for the cigarette than for any NP and were lowest for the NP with the lowest nicotine content.

Conclusions This study provides important insight into nicotine pharmacokinetics and subjective effects during NP use, and demonstrates that NPs can provide nicotine in amounts sufficient to replicate cigarette smokers' nicotine uptake following a switch from conventional cigarettes to these potentially less harmful NP products. Further studies are required to ascertain how physical characteristics of NPs other than nicotine content may affect nicotine delivery, pharmacokinetics and subjective responses.
\end{abstract}

ISRCTN Clinical Trial Registry ISRCTN17828518.

\section{Introduction}

Smoking is a leading cause of numerous human diseases, including lung cancer, chronic obstructive pulmonary disease and cardiovascular disease [1]. The addictive properties of cigarette smoking are primarily due to nicotine, a

Michael McEwan

mike_mcewan@bat.com

1 British American Tobacco (Investments) Limited, Research and Development, Regents Park Road, Southampton SO15 8TL, UK

2 whatIF? Consulting Ltd, Harwell, UK

3 RAI Services Company, 950 Reynolds Blvd., Winston-Salem 27105, NC, USA chemical found naturally in tobacco leaf that transfers into cigarette smoke [2]. Nicotine is absorbed rapidly into the bloodstream during cigarette smoking [3], from where it is distributed throughout the body, causing both systemic and central nervous system effects. In the brain, nicotine acts on neuronal nicotinic receptors, and this interaction may underpin its effects on mood and relaxation [4]. The pharmacokinetic profile of nicotine during cigarette smoking is a rapid rise and fall in plasma nicotine concentrations [5]. Correspondingly, the delivery of nicotine to the brain and the consequent pleasurable effects experienced by the smoker are also rapid [4].

It is now generally accepted that the harmful effects of cigarette smoking are caused by exposure to some of the 8700 or more identified chemical constituents [6-11], some of which contribute to disease development. These effects 


\section{Key Points}

Nicotine pharmacokinetics and subjective effects during the use of a range of NPs were assessed and compared to those during the use of a combustible cigarette.

Nicotine delivery from the NPs was variable, and plasma nicotine concentrations appeared not to be related to the nicotine contents of the individual products, suggesting that physical properties of the NPs other than nicotine content are important for nicotine delivery.

Liking and intent-to-use-again scores were higher for the cigarette than for any NP and were lowest for the NP with the lowest nicotine content.

NPs can provide nicotine in amounts sufficient to replicate cigarette smokers' nicotine uptake following a switch from conventional cigarettes to these potentially less harmful products.

are not substantially caused by the addictive compound nicotine, which is considered by some prominent regulatory and healthcare bodies to be relatively harmless at the levels of uptake during cigarette smoking $[12,13]$. The health risks associated with cigarette smoking are correlated with duration of smoking and degree of daily cigarette consumption $[8,14]$. Epidemiological studies have confirmed that smoking cessation substantially reduces the relative risks of developing smoking-related diseases. In order to increase the number of smokers who would otherwise continue to smoke but switch completely to products with reduced risk potential, a tobacco harm reduction approach has been proposed $[9,11]$, whereby consumers are given access to alternative nicotine products that deliver nicotine without posing the risks associated with tobacco combustion and the inhalation of cigarette smoke toxicants [15].

In the past decade, alternative nicotine delivery products such as e-cigarettes or tobacco heating products have been developed, and it has been proposed that smokers who switch completely to these products instead of continuing to smoke could potentially reduce their relative risks of disease $[16,17]$. More recently, a novel category of modern oral nicotine pouches (NPs) has emerged. NPs are similar in physical form to Swedish snus, which is a pouched smokeless tobacco product that is placed between the upper lip and gum, where nicotine is absorbed through the oral mucosa. Swedish snus is one of the few products recognised by the US FDA as a contributor to tobacco harm reduction [18].
Like snus, NPs are placed between the user's upper lip and the gum [19], where the nicotine released is absorbed into the blood through the oral mucosa. Unlike snus, however, NPs typically do not contain tobacco but pharmaceutical grade nicotine absorbed onto a cellulose matrix in a porous pouch [19-21]. The comparatively simple composition of NPs relative to snus is reflected in a recent toxicant analysis, which showed that NPs contain lower levels than snus of 10 harmful and potentially harmful tobacco constituents (HPHCs) and undetectable levels of a further 13 HPHCs [20]. In vitro studies also indicate that extracts from NPs are significantly less biologically active than extracts from Swedish snus or cigarette smoke [22], supporting a potential role of NPs in tobacco harm reduction. At present, however, there is little information concerning the ability of NPs to deliver nicotine to users or the impact the physical attributes of NPs, such as nicotine content, have on nicotine delivery and pharmacokinetics. Given the importance of nicotine delivery from alternative products in providing smokers with a satisfactory alternative to cigarette smoking [13, 23], studies assessing the delivery and pharmacokinetics of nicotine from NPs are required to inform our understanding of their harm reduction potential.

The primary objectives of the present study were to determine the pharmacokinetics of nicotine absorption into the blood of subjects from different smokeless NP variants as well as a combustible cigarette, and to compare the nicotine delivery from these NPs to one another and to that from a combustible cigarette. We report data for five different NP brands, each of which were mint flavoured but differed in nicotine strength and other physical characteristics. We discuss the implications of our findings regarding the potential of NPs to contribute to tobacco harm reduction by providing smokers with satisfactory alternatives to cigarette smoking.

\section{Methods}

\subsection{Study Design}

This was a randomised, controlled, crossover clinical study carried out at a single clinical site in Uppsala, Sweden. The study was registered in the ISRCTN registry (ISRCTN17828518). Approval was given by an independent ethics committee (Central Swedish Ethics Committee, Uppsala, Sweden; reference number 2019-06294) prior to study commencement. The study was conducted in compliance with the study protocol, the Declaration of Helsinki, the International Council for Harmonisation (ICH) Guideline E6 for Good Clinical Practice (GCP), the Food and Drug Administration (FDA) GCP Code of Federal Regulations Title 21 (part 56) and the European regulation EU 536/2014, including regulations relating to the protection of subjects' 
personal data. Written informed consent was obtained from all subjects prior to participation in the study and before undergoing any study procedures, including screening assessments.

\subsection{Participants}

Among potential recruits attending a screening visit, 35 healthy male or female subjects who met the inclusion criteria were enrolled in the study. Participants were aged 19-55 years with no clinically relevant abnormal findings on physical examination, vital signs assessment, electrocardiogram, clinical laboratory evaluations, oral mucosa examination or in their medical history. All participants were current daily snus users who had regularly used snus products (pouch weight $\geq 0.8 \mathrm{~g}$, containing $\geq 8 \mathrm{mg}$ nicotine) under their upper lip for at least 6 months. They were also current smokers of $\geq 5$ cigarettes per week who had been smoking for at least a year prior to the study. Women of childbearing age were required to use two accepted forms of contraception for approximately 1 month before and after the study.

The main exclusion criteria were pregnancy or breastfeeding (women); self-reported non-inhalation of cigarettes; presence of dental work (including missing molars) that might affect the conduct of the study; presence or history of significant oral and/or pharyngeal inflammation, oral lesions and/or gum disease or temporomandibular joint dysfunction; history of significant hypersensitivity to any excipients of the study product formulations or severe hypersensitivity to any drugs; and the donation of $\geq 400 \mathrm{~mL}$ of blood within 90 days, plasma within 7 days, or platelets within 6 weeks before the study. In addition, individuals who reported the postponement of a decision to quit using tobacco- or nicotine-containing products in order to participate in the study or who had attempted to quit using tobacco- or nicotinecontaining products within the 28 days before the study were also excluded.

All subjects were informed that they were free to quit smoking and withdraw from the study or to withdraw their consent to participate at any time. All subjects were able to ask for advice to stop using tobacco/nicotine products and were to be provided with a smoking cessation helpline number and/or web address for appropriate smoking cessation services if required.

\subsection{Investigational Products}

The following products were examined in this study: (1) a combustible cigarette (Pall Mall Red, BAT; $0.66 \mathrm{mg}$ ISO nicotine yield); (2) Lyft mint NP (BAT; $10 \mathrm{mg}$ nicotine/ pouch); (3) Zyn (Wet) spearmint NP (Swedish Match; $10 \mathrm{mg}$ nicotine/pouch); (4) Nordic Spirit mint NP (Japan Tobacco International; 9 mg nicotine/pouch); (5) Skruf Super White
Fresh Stark mint NP (Imperial Brands; $8 \mathrm{mg}$ nicotine/ pouch); and (6) On! mint NP (Altria; $6 \mathrm{mg}$ nicotine/pouch). The five NPs were commercially available or a commercial specification product (Lyft) at the time of the study.

\subsection{Study Procedures}

At screening, subjects underwent testing to ensure that they met all inclusion and no exclusion criteria, and they completed a smoking/smokeless product use history questionnaire. They were admitted to the clinic site in the afternoon of the day before the first study product use session (Day $-1)$ and were confined to the site until the last assessments at the end of the study (i.e. for a period of $\sim 8$ days). On admission to the clinic site, subjects underwent an eligibility criteria review, vital signs assessments, a symptom-oriented physical examination if indicated, and an oral mucosa examination. At the end of the study, they underwent clinical laboratory testing, an oral mucosa examination, and a physical examination if indicated. After using at least four of the study products, subjects were given the option to go home for 1 day, returning to the clinic the following day to complete their product use sessions.

On admission on Day -1 , subjects were given two of the study NPs to try in order to familiarise themselves with NPs and ensure their tolerance of these study products. A minimum familiarisation period of $30 \mathrm{~min}$ per product was stipulated, with a gap of 1-2 $\mathrm{h}$ between the use of each product.

Before each study day, subjects were required to abstain from using any nicotine product for a period of at least $12 \mathrm{~h}$ before their product use session the following day. On each study day, subjects consumed a light breakfast at least $1 \mathrm{~h}$ before product use and ate a standardised lunch after the 6-h blood sample was drawn. Product use sessions were conducted in the morning of each study day.

On each study day, subjects used a single study product in accordance with a randomisation schedule. For the NPs, subjects were instructed to place the pouch between their top lip and their gum for $60 \mathrm{~min}$. At the end of the 60-min period, they removed the pouch from their mouth. For the combustible cigarette, subjects were asked to smoke a single cigarette ad libitum (i.e. they were not instructed how to puff on the cigarette) for a 5-min period. If the cigarette was still lit at the end of the 5-min period, no further puffing was allowed. If the subject reached the end of the cigarette before the $5 \mathrm{~min}$ had elapsed, no further cigarettes were allowed to be smoked. The number of puffs taken was counted by clinic staff. Subjects were allowed to use their own brand snus or NPs after completion of the product use and blood sampling session until $12 \mathrm{~h}$ prior to the next product use session. During this period, the subjects were only allowed to use their own products, which were dispensed by the clinic staff on request. 


\subsection{Blood Sampling for Nicotine Pharmacokinetics}

Venous blood samples were collected from an indwelling cannula inserted into a forearm vein at the start of each study day. Blood samples $(4 \mathrm{~mL})$ were taken at $-5,3,5,7,10$, $20,45,60,65,75,120,240$ and 360 min relative to the start of each product use session. Blood was collected into a $\mathrm{K}_{2}$-EDTA vacutainer tube. After collection, blood samples were mixed by inverting the tube 10 times to ensure anticoagulation. Samples were then held at $4 \pm 4{ }^{\circ} \mathrm{C}$ until centrifugation at $1900 \pm 38 \mathrm{~g}$ at $4 \pm 4^{\circ} \mathrm{C}$ for $10 \mathrm{~min}$. The time between blood collection and placement in the centrifuge did not exceed $60 \mathrm{~min}$. After centrifugation, the blood plasma fraction was separated into two aliquots of approximately $0.7 \mathrm{~mL}$ each. After separation, plasma was stored at $-20 \pm 5{ }^{\circ} \mathrm{C}$ at the clinical site until shipment to the bioanalytical laboratory. The time between the end of centrifugation and aliquot storage did not exceed $180 \mathrm{~min}$. Plasma nicotine analysis was performed by Anapharm Bioanalytics, Barcelona, Spain. Nicotine was extracted from each sample (study sample, calibration standard or quality control) using a protein precipitation procedure. Briefly, an internal standard (Nicotine-D4) was added to tubes containing plasma, followed by extraction with acetonitrile. Processed samples were stored at $4{ }^{\circ} \mathrm{C}$ until analysis. Samples were analysed using an ACQUITY UPLC ${ }^{\circledR}$ I-Class system (Waters) equipped with an ACUITY UPLC ${ }^{\circledR}$ Binary Solvent Manager, Sample Manager with Flow-Through Needle, Sample Organizer and BEH C18 column (Waters), which was coupled to a QTRAP 6500 mass spectrometer (Sciex).

The mobile phase was an isocratic water/acetonitrile (10/90) mixture with $2.5 \mathrm{mM}$ ammonium acetate at $\mathrm{pH} 5$. The flow rate was $0.50 \mathrm{~mL} / \mathrm{min}$ and the injection volume was $10 \mu \mathrm{L}$. The calibration range was $0.40-39.92 \mathrm{ng} / \mathrm{mL}$, with a lower limit of quantitation (LLOQ) of $0.4 \mathrm{ng} / \mathrm{mL}$.

\subsection{Subjective Effects Assessments}

At the end of the product usage period, subjects completed a product liking questionnaire and an intent-to-use-again questionnaire to evaluate the subjective effects of study product use. Answers were given on a scale of 0 (strongly dislike/ not at all) to 10 (strongly like/very much).

\subsection{Safety Assessments}

Adverse events (AEs) — defined as any untoward medical occurrences in the subjects, including a clinically significant abnormal clinical laboratory finding, symptom or disease temporally associated with the use of a study product (whether or not related to the use of the product) - were selfreported by subjects during the study, either spontaneously or when asked through open-ended questioning by study staff. A product use-emergent AE (PEAE) was defined as an $\mathrm{AE}$ not present prior to the use of a study product or an $\mathrm{AE}$ already present that worsened in intensity or frequency following the use of a study product. All AEs reported following the use of a study product were considered PEAEs. Each PEAE was assigned to the last product used by the subject, with the assignment performed irrespective of any determination of the relationship between the PEAE and product use. A serious adverse event (SAE) was defined as any untoward medical occurrence that results in death, is life-threatening, requires inpatient hospitalisation or prolongation of existing hospitalisation, results in persistent or significant disability or incapacity (defined as a substantial disruption of a person's ability to conduct normal life functions), is a congenital anomaly or birth defect, or is an important medical event (including development of drug dependence or drug abuse) that may jeopardise the subject or may require intervention to prevent one of the other outcomes listed.

\subsection{Sample Size and Statistical Methods}

Based on two-sided pairwise comparisons, and using an eight-product and eight-sequence Williams design, we calculated that a minimum of 32 subjects (4 subjects per sequence) would be necessary to achieve a balanced design and a power of 0.825 with an $\alpha$-level of 0.05 . This would enable us to detect a difference of $20 \%$ in the log scale with an intrasubject coefficient of variation (CV) of 0.3 .

Individual raw nicotine concentration data and derived concentrations were calculated using Phoenix ${ }^{\circledR}$ WinNonlin ${ }^{\circledR}$ version 8.1 (Certara, Princeton, NJ, USA). The pharmacokinetic parameters maximum plasma concentration $\left(C_{\max }\right)$, area under the plasma concentration-time curve between 0 and $6 \mathrm{~h}\left(\mathrm{AUC}_{0-6 \mathrm{~h}}\right)$ and time to reach $C_{\max }\left(T_{\max }\right)$ were calculated using non-compartmental procedures. Reported plasma nicotine concentrations and pharmacokinetic parameters were not corrected for baseline plasma nicotine concentrations; a sensitivity analysis comparing uncorrected and corrected concentrations showed no differences in statistical outcomes. Samples with nicotine concentration values that were below the LLOQ that preceded the first quantifiable concentration were assigned a value of zero for linear plots and for all calculations and summary statistics, but were excluded from semi-logarithmic plots. All other nicotine concentrations below the LLOQ were treated as missing for all analyses. For samples above the upper limit of quantitation (ULOQ), a fourfold dilution factor was applied, and the samples were reanalysed, along with low dilution quality controls.

Descriptive statistics were calculated for nicotine concentrations at each individual time point and for all pharmacokinetic parameters. Individual concentrations, actual sampling 
times and pharmacokinetic parameters were summarised per study product by the following descriptive statistics: number of observations $(\mathrm{N})$, minimum, arithmetic mean, geometric mean, median, maximum, standard deviation (SD) and $\mathrm{CV}$. The geometric mean nicotine $C_{\max }$ and $\mathrm{AUC}_{0-6 \mathrm{~h}}$ were compared among the study products using a mixed-effects analysis of variance (ANOVA) model with treatments, periods and sequences as fixed effects and subjects nested within sequences as random effects. Product comparisons were conducted by using the fitted model with an adjusted significance level of 0.025. Data were analysed on a natural $\log (\ln )$-transformed scale.

Statistical analysis was performed with $\mathrm{SAS}{ }^{\circledR}$ version 9.4 for Windows (SAS Institute Inc., Cary, NC, USA). The reported data correspond to the pharmacokinetic subject set, which was a subset of the full analysis set and excluded subjects who had relevant protocol violations (or circumstances) that affected the evaluation of pharmacokinetic parameters. One protocol deviation occurred due to problems inserting the cannula into a study subject on a single study day when using an oral NP. Consequently, all blood samples after 3 min were missing. A further subject had three missing consecutive blood draws due to an issue with early blood sampling during their combustible cigarette smoking period.

Subjective measures data were summarised by product with frequencies $(n, \%)$ using the original rating $(0-10)$ and a three-category rating (below neutral, neutral, and above neutral, where an original rating of 5 was regarded as neutral). Post-hoc statistical analysis was performed on the original ratings using ANOVA with post-hoc Tukey adjustments.

The randomisation code was not available to the personnel of the bioanalytical facility until the bioanalytical tables had been finalised and audited by the quality assurance department. The statistician and pharmacokineticist were also blind to the study product used on each study day until the final analyses had been performed.

\section{Results}

\subsection{Participant Demographics}

Of 56 subjects who were screened, 35 met the eligibility requirements and were enrolled in the study. There were no withdrawals during the study, and none of the subjects utilised the opportunity to take a 1-day break after receiving at least four study products. Basic subject demographic details are presented in Table 1; the overall gender split was 77:23 males to females, and participants were predominantly white (94\%). From the tobacco use history information reported at screening, all participants reported being current smokers of factory-manufactured cigarettes, and $3(8.6 \%)$ also reported that they currently smoked self-rolled cigarettes. No participants reported that they currently used e-cigarettes, and $9(25.7 \%)$ reported being former e-cigarette users; the remaining $26(74.3 \%)$ participants reported that they had never used e-cigarettes. None of the participants had ever used tobacco heating products.

\subsection{Nicotine Pharmacokinetics}

Mean plasma nicotine concentration-time curves for the NPs and combustible cigarette are shown in Fig. 1, while data for the pharmacokinetic parameters are presented in Table 2.

For the plasma nicotine analysis, the linearity for all runs was $r \geq 0.9981$. Between-run accuracy ranged from 98.71 to $105.97 \%$ of the nominal concentration, and the betweenrun CV was $1.90-4.90 \%$. The within-run accuracy ranged from 98.22 to $102.93 \%$ of the nominal concentration, and the within-run CV was $0.55-3.26 \%$. Analyte recoveries for the low-, medium-, and high-quality control samples were $98.04,99.00$, and $100.62 \%$, respectively, and the recovery of the internal standard was $100.56 \%$.

For each study product, plasma nicotine concentrations rose during use and reached a peak $\left(T_{\max }\right)$ shortly after the end of the 5-min smoking session for the combustible cigarette (median $T_{\max } 7 \mathrm{~min}$ ) or the 60 -min use session for the NPs (median $T_{\max } 60-65 \mathrm{~min}$ ). The geometric mean (CV) $C_{\max }$ for the NPs ranged from 11.9 (26.8) to $18.4(30.1) \mathrm{ng} /$ $\mathrm{mL}$, as compared with $13.9(82.7) \mathrm{ng} / \mathrm{mL}$ for the cigarette, while the geometric mean $(\mathrm{CV}) \mathrm{AUC}_{0-6 \mathrm{~h}}$ ranged from 35.8 (30.6) to 53.7 (27.2) $\mathrm{ng} \cdot \mathrm{h} / \mathrm{mL}$ for the NPs as compared with $25.2(60.1) \mathrm{ng} \cdot \mathrm{h} / \mathrm{mL}$ for the cigarette. The mean elimination half-life $\left(T_{1 / 2}\right)$ for the plasma nicotine decay $\pm \mathrm{SD}$ was

Table 1 Demographic data for the study subjects collected at screening

\begin{tabular}{lrrrr}
\hline Variable & $n$ & $\%$ & Mean (SD) & Median (range) \\
\hline Age (years) & 35 & $27.9(8.1)$ & $26.0(19.0-55.0)$ \\
Weight $(\mathrm{kg})$ & 35 & & $75.9(12.5)$ & $76.0(57.0-104.0)$ \\
Height $(\mathrm{cm})$ & 35 & $180.3(8.4)$ & $179.0(161.0-195)$ \\
Body mass index (kg/ & 35 & $23.3(3.2)$ & $22.6(22.6-29.8)$ \\
$\left.\quad \mathrm{m}^{2}\right)$ & & & & \\
Sex & & & & \\
$\quad$ Male & 27 & 77.1 & & \\
Female & 8 & 22.9 & & \\
Race & & & & \\
$\quad$ White & 33 & 94.3 & & \\
$\quad$ Black/African Ameri- & 1 & 2.9 & & \\
$\quad$ can & & & & \\
$\quad$ Asian & 1 & 2.9 & & \\
Ethnicity & & & & \\
$\quad$ Hispanic or Latino & 2 & 5.7 & & \\
$\quad$ Not Hispanic or Latino & 33 & 94.3 & & \\
\hline
\end{tabular}


similar for all study products, ranging from $2.15 \pm 0.31 \mathrm{~h}$ for the $6 \mathrm{mg}$ On! NP to $2.82 \pm 3.29 \mathrm{~h}$ for the $10 \mathrm{mg}$ Zyn NP. However, it should be noted that there was more variability in the $T_{1 / 2}$ for Zyn, based on an inexplicably high result from one subject.

We assessed differences in pharmacokinetic parameters among the six study products using the BAT commercial specification Lyft $10 \mathrm{mg}$ NP as a reference (Table 3). $C_{\max }$ and $\mathrm{AUC}_{0-6 \mathrm{~h}}$ were significantly greater for the $\mathrm{Lyft}$ $10 \mathrm{mg}$ NP than for the combustible cigarette $(p<0.0084$ and $p<0.0001$, respectively). In addition, $C_{\max }$ and AUC 0-6h were significantly higher (both $p<0.0001$ ) for the Lyft $10 \mathrm{mg}$ NP as compared with the Zyn $10 \mathrm{mg}$ NP. Similarly, $C_{\max }$ and $\mathrm{AUC}_{0-6 \mathrm{~h}}$ were significantly higher $(p=0.0005$ and $p<0.0001$, respectively) for the Lyft $10 \mathrm{mg} \mathrm{NP}$ than for the Skruf $8 \mathrm{mg}$ NP. No significant difference in $C_{\max }$ and $\mathrm{AUC}_{0-6 \mathrm{~h}}$ between the Lyft $10 \mathrm{mg}$ NP and the other NPs was observed. $T_{\max }$ was higher for all NPs (60-65 min) than for the cigarette ( $7 \mathrm{~min})$.

Further to this, we investigated relative bioavailability by dividing the $C_{\max }$ of each of the products by their respective nominal nicotine concentration in unused pouches. Relative
Fig. 1 Plasma nicotine concentrations over time. Each point shows the mean plasma nicotine concentration for all subjects in the pharmacokinetic population $(n=35)$. Errors bars correspond to $\mathrm{SD}$

Table 2 Summary of nicotine pharmacokinetic parameters

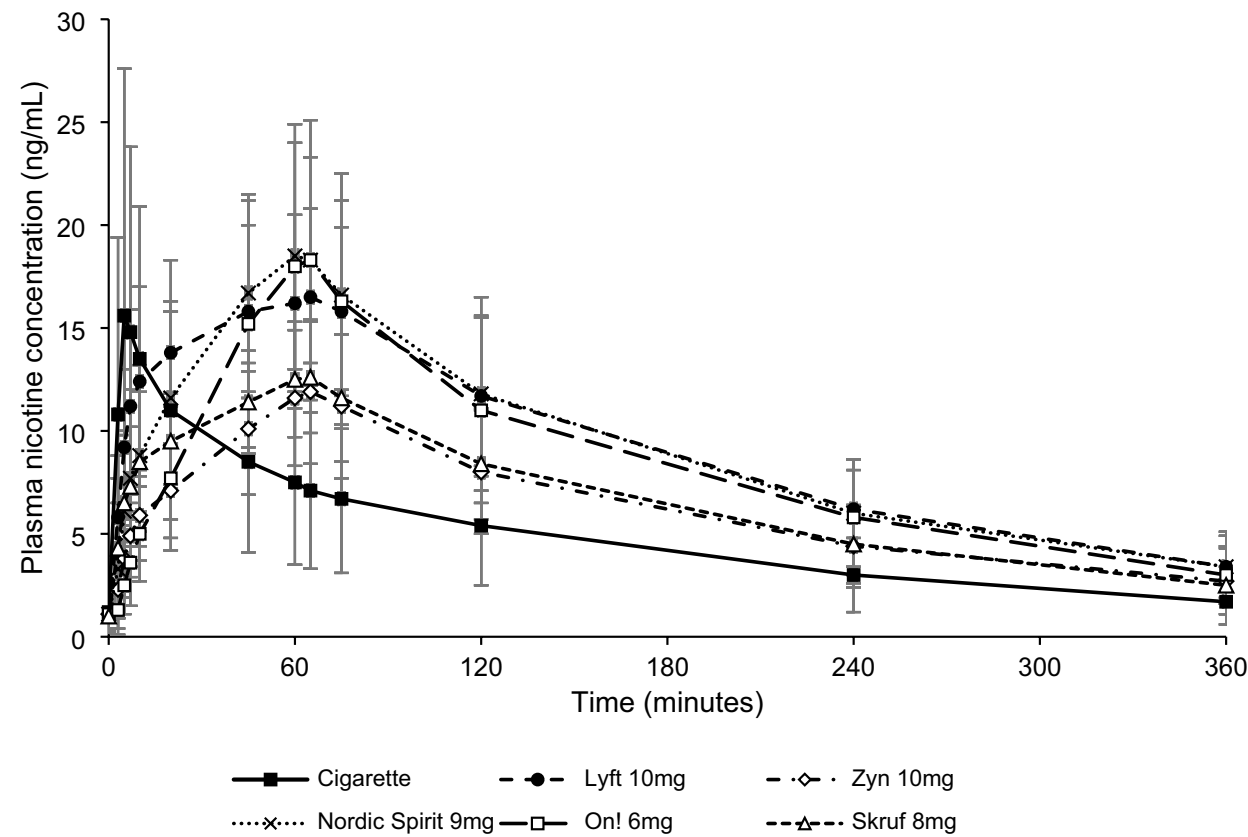

\begin{tabular}{llllll}
\hline Product & $\begin{array}{l}\text { Nicotine/ } \\
\text { pouch }(\mathrm{mg})\end{array}$ & $C_{\max }(\mathrm{ng} / \mathrm{mL})$ & $\mathrm{AUC}_{0-6 \mathrm{~h}}(\mathrm{ng} \cdot \mathrm{h} / \mathrm{mL})$ & $T_{\max }(\mathrm{min})$ & $T_{1 / 2}(\mathrm{~h})^{\mathrm{a}}$ \\
\hline Cigarette & N/A & $\begin{array}{l}13.9(82.7) \\
n=34\end{array}$ & $\begin{array}{l}25.2(60.1) \\
n=34\end{array}$ & $\begin{array}{l}7(3,20) \\
n=34\end{array}$ & $\begin{array}{l}2.3(0.49) \\
n=32\end{array}$ \\
& & $17.1(24.0)$ & $53.7(27.2)$ & $60(7,76)$ & $2.2(0.48)$ \\
Lyft NP & 10 & $n=35$ & $n=35$ & $n=35$ & $n=35$ \\
& & $11.9(26.8)$ & $35.8(30.6)$ & $65(45,75)$ & $2.8(3.29)$ \\
Zyn NP & 10 & $n=35$ & $n=35$ & $n=35$ & $n=34$ \\
& & $18.4(30.1)$ & $52.8(30.5)$ & $62(10,120)$ & $2.2(0.42)$ \\
Nordic Spirit NP & 9 & $n=35$ & $n=35$ & $n=35$ & $n=33$ \\
& & $17.5(43.8)$ & $46.9(44.2)$ & $65(45,75)$ & $2.2(0.31)$ \\
On! NP & 6 & $n=35$ & $n=35$ & $n=35$ & $n=34$ \\
& \multirow{2}{*}{$\begin{array}{l}* \\
\text { Skruf NP }\end{array}$} & $13.0(20.2)$ & $39.0(26.4)$ & $60(3,75)$ & $2.2(0.42)$ \\
& & $n=35$ & $n=35$ & $n=35$ & $n=34$ \\
\hline
\end{tabular}

Data are presented as geometric mean (geometric coefficient of variation) for $C_{\max }$ and $\mathrm{AUC}_{0-6 \mathrm{~h}}$; median (min, $\max$ ) for $T_{\max }$; and mean (SD) for $T_{1 / 2}$

N/A not applicable, $C_{\max }$ maximum plasma nicotine concentration, $A U C_{0-6 h}$ area under the plasma nicotine concentration-time curve between 0 and $6 \mathrm{~h}, T_{\max }$ time of maximum plasma nicotine concentration, $T_{1 / 2}$ half-life of plasma nicotine decay, $n$ number of subjects

${ }^{a}$ Eight study periods were excluded for $T_{1 / 2}$ due to the lack of a linear fit in the elimination phase 
bioavailability for each product was calculated against the product with the lowest level, which was Zyn (relative bioavailability 1.0). Skruf was the next highest and was 1.37 times higher than Zyn, Lyft was 1.44 times higher, and then Nordic Spirit and On! were 1.72 and 2.45 times higher than Zyn, respectively.

\subsection{Subjective Effects}

Data from subjective effects assessments are presented in Table 4. Broadly speaking, scores for product liking and intent to use the product again were highest for the combustible cigarette; that is, more participants reported an intent to use the product again (i.e. a score $>5$ ) for the cigarette $(n=21)$ than for the NPs $(n=14-19)$.

Both the product liking and the intent to use the product again scores were lowest (i.e. the greatest number of participants reported a score of $<5$ ) for the NP with the lowest nicotine content ( $6 \mathrm{mg}$ On! NP). On! showed significantly lower scores for product liking than the combustible cigarette

Table 3 Statistical comparisons for nicotine pharmacokinetic parameters

\begin{tabular}{llllll}
\hline Comparison & & & & $p$ value & \\
\cline { 1 - 1 } \cline { 5 - 6 } \cline { 5 - 6 } Reference product & Comparator product & & $\mathrm{AUC}_{0-6 \mathrm{~h}}$ & $C_{\max }$ \\
\hline Cigarette & Lyft NP (10 mg) & & 0.0001 & 0.0084 \\
Lyft NP (10 mg) & Zyn NP (10 mg) & & 0.0001 & 0.0001 \\
Lyft NP (10 mg) & Nordic Spirit NP (9 mg) & & 0.8257 & 0.3086 \\
Lyft NP (10 mg) & On! NP (6 mg) & & 0.0265 & 0.7498 \\
Lyft NP (10 mg) & Skruf NP (8 mg) & & 0.0001 & 0.0005 \\
\hline
\end{tabular}

Data are unadjusted two-sided $p$ values for the comparison

$C_{\max }$ maximum plasma nicotine concentration, $\mathrm{AUC}_{0-6 h}$ area under the plasma nicotine concentration-time curve between 0 and $6 \mathrm{~h}$ $(p=0.0216)$ and the Zyn product $(p=0.0392)$. In addition to this, On! showed significantly lower scores for intent to use again than the combustible cigarette $(p=0.0030)$ and the Lyft product $(p=0.0363)$. There were no further significant differences between the products in the subjective effects scores.

\subsection{Adverse Events}

In total, 27 PEAEs were reported by study participants. The most commonly affected system organ classes were "nervous system disorders" (10 PEAEs reported, including dizziness, headache and restless legs syndrome) and "general disorders" (5 PEAEs reported, including asthenia, catheter site pain and fatigue). Most (26/27) PEAEs were mild in intensity, one was moderate in intensity, and none were severe in intensity. Of the 27 PEAEs reported, 19 of the PEAEs were judged to be related to the study products by the study principal investigator or designee. Of these, three were related to the cigarette while the rest were evenly spread among the NPs. Almost all AEs related to NP use were mild, apart from one which was moderate in intensity. No serious AEs or withdrawals due to adverse events occurred in the study.

\section{Discussion}

In this paper, we have reported data from a randomised crossover study examining nicotine pharmacokinetics and subjective effects in dual snus and cigarette consumers who used five different NPs and a combustible cigarette under defined conditions. We found that the increase in nicotine blood plasma concentration from NP use was slower (i.e. a longer $T_{\max }$ ) than that seen when participants smoked combustible cigarettes, whereas the amount of nicotine blood plasma concentration (i.e. $C_{\max }$ and $\mathrm{AUC}_{0-6 \mathrm{~h}}$ ) was greater
Table 4 Summary of subjective effects measures

\begin{tabular}{|c|c|c|c|c|c|c|}
\hline Question & Cigarette & Lyft 10 mg NP & Zyn 10 mg NP & $\begin{array}{l}\text { Nordic } \\
\text { Spirit } 9 \text { mg } \\
\text { NP }\end{array}$ & On! 6 mg NP & Skruf $8 \mathrm{mg}$ NP \\
\hline \multicolumn{7}{|c|}{ At this moment, how much do you like the product? ${ }^{\mathrm{a}}$} \\
\hline$<5$ & $8(22.9)$ & $8(22.9)$ & $7(20.0)$ & $13(37.1)$ & $21(60.0)$ & $9(25.7)$ \\
\hline 5 & $6(17.1)$ & $11(31.4)$ & $9(25.7)$ & $7(20.0)$ & $4(11.4)$ & $12(34.3)$ \\
\hline$>5$ & $21(60.0)$ & $16(45.7)$ & $19(54.3)$ & $15(42.9)$ & $10(28.6)$ & $14(40.0)$ \\
\hline \multicolumn{7}{|c|}{ Rate the degree to which you would like to use the product again ${ }^{\mathrm{b}}$} \\
\hline$<5$ & $11(31.4)$ & $15(42.9)$ & $20(57.1)$ & $21(60.0)$ & $27(77.1)$ & $20(57.1)$ \\
\hline 5 & $4(11.4)$ & 8 (22.9) & $2(5.7)$ & $3(8.6)$ & $3(8.6)$ & $5(14.3)$ \\
\hline$>5$ & $20(57.1)$ & $12(34.3)$ & $13(37.1)$ & $11(31.4)$ & $5(14.3)$ & $10(28.6)$ \\
\hline
\end{tabular}

Data are presented as $n(\%)$. Number of subjects (denominator) $=35$ in each case

${ }^{a}$ Original scale was from 0 (strongly dislike) to 10 (strongly like)

${ }^{\mathrm{b}}$ Original scale was from 0 (not at all) to 10 (very much) 
or similar to that seen with cigarette smoking for the NPs examined. Lastly, the subjective questionnaire demonstrated that product liking and intent-to-use-again scores were greater for the higher nicotine content NPs, although these assessments of liking and intent were lower for all NPs than for combustible cigarettes.

Although $C_{\max }$ and $\mathrm{AUC}_{0-6 \mathrm{~h}}$ were greater for some of the NPs than for the cigarette, we note that the 60 -min period of use applied in this study is typically higher than the manufacturers' recommended usage time for these products. Commonly, manufacturers recommend a usage time of approximately $30 \mathrm{~min}$, in which case the "real-world" nicotine $C_{\max }$ and $\mathrm{AUC}_{0-6 \mathrm{~h}}$ for NPs are likely to be lower than the values observed in this study. In this study, a 60-min usage time was selected based on prior snus pouch nicotine pharmacokinetic studies that had a 60-min mouth hold time based on product use data $[24,25]$. At present, there are no other data comparing nicotine pharmacokinetics between cigarettes and this relatively new product category of NPs, but previous studies have similarly reported greater AUC and $C_{\max }$ values for some types of snus than for cigarettes [25-27], probably due to the longer period of snus use relative to smoking a single cigarette.

In addition to usage time, the actual nicotine blood plasma concentration will be influenced by various factors, including the nicotine content of the product, the amount of nicotine extracted during use, average daily consumption (ADC), use behaviour and individual metabolism. In particular, ADC is an important factor in daily nicotine exposure and blood plasma concentration. A recent study reported an ADC of 8.6 pouches/day among solus NP users as compared with an ADC of $\sim 14$ cigarettes/day among smokers [20]. However, the purpose of the present study was to compare different NPs and a cigarette. To achieve this, we measured the nicotine pharmacokinetics for the NPs after single product use for a relatively long time, resulting in higher $\mathrm{AUC}_{0-6 \mathrm{~h}}$ values for the NPs than for the cigarette. Further studies are needed to establish the daily nicotine exposure and blood plasma concentrations resulting from NP use, as well as consumer behaviour in using these products, including average use times and ADC.

As yet, there is little information concerning the nicotine pharmacokinetic properties of NPs, their ability to deliver nicotine to users, and the effect of such nicotine delivery on the blood plasma concentration. While many papers have reported such data for snus [25-27] (a product similar in format and usage to NPs but which utilises tobacco as a source of nicotine) as well as nicotine gum [25-31] and other oral nicotine products [31-35], to our knowledge, only one study has reported the nicotine pharmacokinetics for NPs [21]. In that study, Lunell et al. [21] compared the nicotine pharmacokinetics between NPs and snus among snus users, and found that an NP with a nicotine content of $6 \mathrm{mg}$ resulted in greater AUC as compared with Swedish snus with a nicotine content of $8 \mathrm{mg}$, implying that NPs are likely to satisfy the nicotine cravings of snus users. The extraction of nicotine from NPs during use appears to be greater than the extraction of nicotine from snus; $58 \%$ on average over $60 \mathrm{~min}$ [21] compared with $33 \%$ on average from snus [20]. Our study, conducted among current smokers, found that both $C_{\max }$ and $\mathrm{AUC}_{0-6 \mathrm{~h}}$ were generally higher for NPs than for a combustible cigarette, and thus these products may be able to satisfy the nicotine cravings of smokers as part of a tobacco harm reduction approach. However, it should be noted that NPs took a longer time to reach $T_{\max }$, and the higher $C_{\max }$ and $\mathrm{AUC}_{0-6 \mathrm{~h}}$ of NPs should be contextualised with NP use behaviour, as discussed below.

It has been suggested for other types of nicotine delivery products, such as e-cigarettes, that a higher nicotine delivery from these devices (i.e. closer to that of cigarette smoking) is likely to provide smokers with a more satisfactory alternative to conventional cigarettes [23]. This is also the case for nicotine replacement therapy products; medically approved oral smoking cessation aids such as nicotine gum and nicotine lozenges with a higher nicotine content and which give greater nicotine delivery to users [36] have previously been shown to be more effective in assisting smoking cessation than products with a lower nicotine content, particularly in more heavily dependent smokers [37-40]. Given this, the higher $C_{\max }$ and $\mathrm{AUC}_{0-6 \mathrm{~h}}$ of NPs relative to cigarettes observed in the present study suggest that NPs have sufficient nicotine delivery to be a satisfactory substitute product for smokers seeking alternatives to conventional cigarettes, although NPs are neither marketed nor approved for use as aids to quit smoking. We note that the proportion of participants reporting a positive response in the product liking questionnaire was lower for the NPs than for the cigarette, indicating that other factors are also important in providing smokers with satisfactory alternatives to continued combustible cigarette smoking.

An interesting facet of our data is the lack of a relationship between the nicotine contents of the NPs and the pharmacokinetic parameters $C_{\max }$ and AUC, while the existence of such a relationship has been demonstrated for other oral nicotine products such as nicotine gum [30]. Although the NPs used by participants in this study spanned a range of nicotine contents from 6 to $10 \mathrm{mg}$ nicotine per pouch, a higher nicotine content did not necessarily appear to give rise to a higher $C_{\max }$ and $\mathrm{AUC}_{0-6 \mathrm{~h}}$. For example, $C_{\max }$ and $\mathrm{AUC}_{0-6 \mathrm{~h}}$ were highest for the $9 \mathrm{mg}$ Nordic Spirit (18.4 ng/ $\mathrm{mL})$ and $10 \mathrm{mg}$ Lyft $(53.7 \mathrm{ng} \cdot \mathrm{h} / \mathrm{mL}) \mathrm{NPs}$, respectively, but were lowest for the $10 \mathrm{mg}$ Zyn NP $(11.9 \mathrm{ng} / \mathrm{mL}$ and 35.8 $\mathrm{ng} \cdot \mathrm{h} / \mathrm{mL}$, respectively). This is supported by the relative bioavailablity of nicotine from the NPs used in this study, which showed that Zyn had the lowest level, followed by Skruf, Lyft, Nordic Spirit and On!, with the latter three products 
having relative bioavailabilities ranging from 1.37 to 2.45 times higher than Zyn. However, the study products were selected from a similar nicotine concentration category within each brand (i.e. we chose the equivalent 'strong' nicotine concentration of each particular brand), and the various products have different features (e.g. pouch dimensions, $\mathrm{pH}$ and pouch constituents) that may enable the $6 \mathrm{mg}$ product to provide a higher nicotine blood plasma concentration than some products with higher nicotine contents. It is also noteworthy in our study that, while the NP with the lowest nicotine content (6 mg On!) had one of the highest $C_{\max }$ values $(17.5 \mathrm{ng} / \mathrm{mL})$ and the highest nicotine bioavailability, the subjective effects scores (liking and intent to use again) were lowest for this NP, whereas there were no major differences among the other four NP products with differing nicotine contents. Notably, the results for the $10 \mathrm{mg}$ Zyn NP contrast with those published by Lunell et al. [21], who reported similar $C_{\max }(18.5 \mathrm{ng} / \mathrm{mL})$ and $\mathrm{AUC}_{\infty}(58.4 \mathrm{ng} \cdot \mathrm{h} / \mathrm{mL})$ values for an $8 \mathrm{mg} \mathrm{Zyn}$ NP to the highest values observed in this study $\left(C_{\max } 18.4 \mathrm{ng} / \mathrm{mL}\right.$ and $\mathrm{AUC}_{0-6 \mathrm{~h}} / \mathrm{AUC}_{\infty}$ $53.7 / 64.3 \mathrm{ng} \cdot \mathrm{h} / \mathrm{mL}$ ). Furthermore, although the $10 \mathrm{mg} \mathrm{Zyn}$ NP had the lowest $C_{\max }$ and $\mathrm{AUC}_{0-6 \mathrm{~h}}$ values of all the NPs, it was one of the best-performing NPs for the subjective effect liking score. These findings are important because they suggest that physical aspects other than nicotine content (e.g. $\mathrm{pH}$, moisture content, and pouch materials, ingredients and dimensions) may affect both the nicotine pharmacokinetics and subjective effects. Furthermore, our findings suggest that, for each individual product, nicotine pharmacokinetics and subjective effects-and thus the potential impact in providing smokers with satisfactory alternatives to continued cigarette smoking — need to be empirically determined and cannot be assumed from the nicotine content alone.

Nicotine pouches are designed to be placed between the gum and the upper lip such that the nicotine released from the pouch is absorbed through the oral mucosa. However, it is possible that some nicotine may pass into the saliva and be swallowed. In our study, the $T_{1 / 2}$ values for the NPs used in this study ranged from 2.2 to $2.8 \mathrm{~h}$ (Table 2), in line with the reported nicotine plasma elimination half-life [41]. Any absorption of nicotine through the gastrointestinal tract might lead to a second $T_{\max }$ peak at around $2-3 \mathrm{~h}$ according to the $T_{\max }$ of ingested nicotine reported by Dautzenberg et al. [42]. There was no secondary peak detected in this study, which suggests that the nicotine from NPs is predominately absorbed via the oral mucosa (buccal absorption).

This study has some limitations. First, it was carried out among combustible cigarette users in Sweden, and as such our findings may not be generalisable to other cohorts (e.g. current non-smokers using other nicotine products such as snus) or to populations of other countries in which tobacco product use patterns may differ. Second, product use was limited to a single time-controlled use of an NP product on a single day, and as such we cannot infer whether nicotine pharmacokinetics will change over time following continued use or when individuals use NPs for shorter or longer time periods than the 60-min use period stipulated in this study protocol. Third, our cohort comprised current snus users who were also cigarette smokers, and as such our findings do not provide information on nicotine pharmacokinetics and subjective effects of NP use in current non-users of oral tobacco products.

\section{Conclusion}

In conclusion, data from this study provide important insight into nicotine delivery, pharmacokinetics and subjective effects during the use of a range of NPs in current smokers who also use snus, and demonstrate that while the $C_{\max }$ and AUC values from these products match or exceed those of a combustible cigarette during smoking, $T_{\max }$ is slower. Further, we also demonstrate that while liking and intent-to-useagain scores were lower for the studied NPs than for combustible cigarettes, significant numbers of subjects reported positive liking and strong intent-to-use-again scores for NPs. Further studies performed in a more naturalistic setting are required to ascertain whether NPs can offer smokers a satisfactory complete substitute for continued cigarette smoking and to examine how different physical characteristics of NPs other than nicotine content impact nicotine delivery, pharmacokinetics and subjective effects.

Acknowledgements The authors gratefully acknowledge Clinical Trial Consultants AB (Uppsala, Sweden), Oy 4Pharma Ltd (Turku, Finland), 4Pharma AB (Lund, Sweden) and A+ Science (Stockholm, Sweden) in supporting the design, conduct and analysis of this study.

\section{Declarations}

Funding The study was supported by British American Tobacco (Investments) Limited, the manufacturer of Lyft and Velo smokeless oral nicotine pouches.

Competing interests All authors are current employees of British American Tobacco (Investments) Limited except JM who is an employee of RAI Services Company and IMF who is a consultant contracted by British American Tobacco (Investments) Limited.

Ethics approval The study was conducted in compliance with the study protocol, the Declaration of Helsinki, the International Council for Harmonisation (ICH) Guideline E6 for Good Clinical Practice (GCP), the Food and Drug Administration (FDA) GCP Code of Federal Regulations Title 21 (part 56) and the European regulation EU 536/2014, including regulations relating to the protection of subjects' personal data. Approval was given by an independent ethics committee (Central Swedish Ethics Committee, Uppsala, Sweden; reference number 201906294) prior to study commencement. 
Informed consent Written informed consent was obtained from all subjects prior to participation in the study and before undergoing any study procedures, including screening assessments.

Consent for publication Patients signed an informed consent regarding the publishing of their data.

Availability of data and materials Data for this study are available on request.

Code availability Code for this study are available on request.

Author contributions MM was the study lead, designed the study and edited the manuscript; DA was study support, designed the study and edited the manuscript; NG and GH assisted with the design of the study; OC assisted with the design of the study and designed the statistical approach; IF wrote the main manuscript text; and JM conceived the study. All authors reviewed the manuscript.

Open Access This article is licensed under a Creative Commons Attribution-NonCommercial 4.0 International License, which permits any non-commercial use, sharing, adaptation, distribution and reproduction in any medium or format, as long as you give appropriate credit to the original author(s) and the source, provide a link to the Creative Commons licence, and indicate if changes were made. The images or other third party material in this article are included in the article's Creative Commons licence, unless indicated otherwise in a credit line to the material. If material is not included in the article's Creative Commons licence and your intended use is not permitted by statutory regulation or exceeds the permitted use, you will need to obtain permission directly from the copyright holder. To view a copy of this licence, visit http://creativecommons.org/licenses/by-nc/4.0/.

\section{References}

1. US Department of Health and Human Services. The health consequences of smoking: 50 years of progress: a report of the Surgeon General. Atlanta: Department of Health and Human Services, Centers for Disease Control and Prevention, National Center for Chronic Disease Prevention and Health Promotion, Office on Smoking and Health; 2014. https://www.ncbi.nlm.nih.gov/ books/NBK179276/pdf/Bookshelf_NBK179276.pdf. Accessed 9 Dec 2021.

2. Hukkanen J, Jacob P 3rd, Benowitz NL. Metabolism and disposition kinetics of nicotine. Pharmacol Rev. 2005;57:79-115.

3. Lunell E, Molander L, Ekberg K, Wahren J. Site of nicotine absorption from a vapour inhaler-comparison with cigarette smoking. Eur J Clin Pharmacol. 2000;55:737-41.

4. Benowitz NL. Clinical pharmacology of nicotine. Annu Rev Med. 1986;37:21-32.

5. Benowitz NL. Pharmacology of nicotine: addiction, smokinginduced disease, and therapeutics. Annu Rev Pharmacol Toxicol. 2009;49:57-71.

6. Farsalinos KE, Le Houezec J. Regulation in the face of uncertainty: the evidence on electronic nicotine delivery systems (e-cigarettes). Risk Manag Healthc Policy. 2015;8:157-67.

7. Food and Drug Administration. Harmful and potentially harmful constituents in tobacco products and tobacco smoke; established list. Docket no. FDA-2012-N-0143. Federal Register 2012;77.

8. International Agency for Research on Cancer. Reversal of risk after quitting smoking. IARC Handbooks of Cancer Prevention, vol. 11. Lyon: IARC; 2007.
9. Institute of Medicine. Clearing the smoke-assessing the science base for tobacco harm reduction. Washington, DC: The National Academies Press; 2001.

10. Rodgman A, Perfetti T. The chemical components of tobacco and tobacco smoke. Boca Raton: CRC Press; 2013.

11. Stratton K, Shetty P, Wallace R, Bondurant S. Clearing the smoke: the science base for tobacco harm reduction-executive summary. Tob Control. 2001;10:189-95.

12. Public Health England. Health matters: stopping smoking — what works?. London: PHE Publications; 2019. https://www.rcplondon. ac.uk/projects/outputs/nicotine-without-smoke-tobacco-harmreduction. Accessed 08 July 2021.

13. Royal College of Physicians. Nicotine without smoke. Tobacco harm reduction. A report by the Tobacco Advisory Group of the Royal College of Physicians. London: Royal College of Physicians; 2016.

14. Doll R, Peto R, Wheatley K, Gray R, Sutherland I. Mortality in relation to smoking: 40 years' observations on male British doctors. Brit Med J. 1994;309:901-11.

15. Gottlieb S, Zeller M. A nicotine-focused framework for public health. N Engl J Med. 2017;377:1111-4.

16. Murphy J, Gaca M, Lowe F, Minet E, Breheny D, Prasad K, Camacho O, Fearon IM, Liu C, Wright C, McAdam K, Proctor C. Assessing modified risk tobacco and nicotine products: description of the scientific framework and assessment of a closed modular electronic cigarette. Regul Toxicol Pharmacol. 2017;90:342-57.

17. McNeill A, Brosen LS, Calder R, Bauld L, Robson D. Evidence review of e-cigarettes and heated tobacco products 2018. A report commissioned by Public Health England. London: PHE Publications; 2018. https://assets.publishing.service.gov.uk/government/ uploads/system/uploads/attachment_data/file/684963/Evidence_ review_of_e-cigarettes_and_heated_tobacco_products_2018.pdf. Accessed 08 Jul 2021.

18. Clarke E, Thompson K, Weaver S, Thompson J, O'Connell G. Snus: a compelling harm reduction alternative to cigarettes. Harm Reduct J. 2019;16:62. https://doi.org/10.1186/s12954-019-0335-1.

19. Plurphanswat N, Hughes JR, Fagerström K, Rodu B. Initial information on a novel nicotine product. Am J Addict. 2020;29:279-86.

20. Azzopardi D, Liu C, Murphy J. Chemical characterization of tobacco-free "modern" oral nicotine pouches and their position on the toxicant and risk continuums. Drug Chem Toxicol. 2021;25:1-9.

21. Lunell E, Fagerström K, Hughes J, Pendrill R. Pharmacokinetic comparison of a novel non-tobacco-based nicotine pouch (ZYN) with conventional, tobacco-based Swedish snus and American moist snuff. Nicotine Tob Res. 2020;22:1757-63.

22. Bishop E, East N, Bozhilova S, Santopietro S, Smart D, Taylor M, Meredith S, Baxter A, Breheny D, Thorne D, Gaca M. An approach for the extract generation and toxicological assessment of tobacco-free 'modern' oral nicotine pouches. Food Chem Toxicol. 2020;145:111713.

23. Hajek P, Pittaccio K, Pesola F, Myers Smith K, Phillips-Waller A, Przulj D. Nicotine delivery and users' reactions to Juul compared with cigarettes and other e-cigarette products. Addiction. 2020;115:1141-8.

24. Digard H, Errington G, Richter A, McAdam K. Patterns and behaviors of snus consumption in Sweden. Nicotine Tob Res. 2009;11:1175-81. https://doi.org/10.1093/ntr/ntp118 (Epub 2009 Aug 17).

25. Digard H, Proctor C, Kulasekaran A, Malmqvist U, Richter A. Determination of nicotine absorption from multiple tobacco products and nicotine gum. Nicotine Tob Res. 2013;15:255-61.

26. Lunell E, Lunell M. Steady-state nicotine plasma levels following use of four different types of Swedish snus compared with 
2-mg Nicorette chewing gum: a crossover study. Nicotine Tob Res. 2005;7:397-403.

27. Lunell E, Curvall M. Nicotine delivery and subjective effects of Swedish portion snus compared with $4 \mathrm{mg}$ nicotine polacrilex chewing gum. Nicotine Tob Res. 2011;13:573-8.

28. Benowitz NL, Hukkanen J, Jacob P 3rd. Nicotine chemistry, metabolism, kinetics and biomarkers. Handb Exp Pharmacol. 2009;192:29-60.

29. Hansson A, Rasmussen T, Kraiczi H. Single-dose and multipledose pharmacokinetics of nicotine $6 \mathrm{mg}$ gum. Nicotine Tob Res. 2017;19:477-83.

30. Leischow SJ, Sachs DP, Hansen MD, Bostrom AG. Nicotine polacrilex dose effects: serum nicotine levels and sensory characteristics. Psychopharmacology. 1995;117:125-9.

31. McRobbie H, Thornley S, Bullen C, Lin RB, Senior H, Laugesen M, Whittaker R, Hajek P. A randomized trial of the effects of two novel nicotine replacement therapies on tobacco withdrawal symptoms and user satisfaction. Addiction. 2010;105:1290-8.

32. Choi JH, Dresler CM, Norton MR, Strahs KR. Pharmacokinetics of a nicotine polacrilex lozenge. Nicotine Tob Res. 2003;5:635-44.

33. Foulds J, Russell MA, Jarvis MJ, Feyerabend C. Nicotine absorption and dependence in unlicensed lozenges available over the counter. Addiction. 1998;93:1427-31.

34. Koszowski B, Viray LC, Stanfill SB, Lisko JG, Rosenberry ZR, Potts JL, Pickworth WB. Nicotine delivery and pharmacologic response from Verve, an oral nicotine delivery product. Pharmacol Biochem Behav. 2015;136:1-6.
35. Kotlyar M, Mendoza-Baumgart MI, Li ZZ, Pentel PR, Barnett BC, Feuer RM, Smith EA, Hatsukami DK. Nicotine pharmacokinetics and subjective effects of three potential reduced exposure products, moist snuff and nicotine lozenge. Tob Control. 2007;16:138-42.

36. McNabb ME, Ebert RV, McCusker K. Plasma nicotine levels produced by chewing nicotine gum. JAMA. 1982;248:865-8.

37. Henningfield JE. Nicotine medications for smoking cessation. N Engl J Med. 1995;333:1196-203.

38. Herrera N, Franco R, Herrera L, Partidas A, Rolando R, Fagerström KO. Nicotine gum, 2 and $4 \mathrm{mg}$, for nicotine dependence. A double-blind placebo-controlled trial within a behavior modification support program. Chest. 1995;108:447-51.

39. Sachs DP. Effectiveness of the 4-mg dose of nicotine polacrilex for the initial treatment of high-dependent smokers. Arch Intern Med. 1995;155:1973-80.

40. Shiffman S, Dresler CM, Hajek P, Gilburt SJ, Targett DA, Strahs KR. Efficacy of a nicotine lozenge for smoking cessation. Arch Intern Med. 2002;162:1267-76.

41. Benowitz NL, Jacob P 3rd. Metabolism of nicotine to cotinine studied by a dual stable isotope method. Clin Pharmacol Ther. 1994;56:483-93. https://doi.org/10.1038/clpt.994.169.

42. Dautzenberg B, Nides M, Kienzler JL, Callens A. Pharmacokinetics, safety and efficacy from randomized controlled trials of 1 and $2 \mathrm{mg}$ nicotine bitartrate lozenges (Nicotinell). BMC Clin Pharmacol. 2007;7:11. https://doi.org/10.1186/472-6904-7-11. 